\title{
Ein Leoparden-Fund, Panthera pardus (L., 1758), aus dem jungpleistozänen Rixdorfer Horizont von Berlin und die Verbreitung des Leoparden im Pleistozän Europas
}

\author{
Karlheinz Fischer ${ }^{1}$ \\ Mit 3 Abbildungen und 1 Tabelle
}

\section{Zusammenfassung}

Erstmals wurde das Vorkommen des Leoparden (Panthera pardus) im Rixdorfer Horizont (Früh-Weichsel, Jungpleistozän) von Berlin-Brandenburg durch ein Humerus-Fragment von Niederlehme bei Königs Wusterhausen nachgewiesen. Es ist das bisher nördlichste Vorkommen des Leoparden in Mitteleuropa.

Schlüsselwörter: Panthera, Humerus, Jungpleistozän, Berlin, Deutschland.

\begin{abstract}
The finding of leopard, Panthera pardus (L., 1758), from the late Pleistocene horizon of Rixdorf in Berlin and the occurrence of leopards in the Pleistocene of Europe are described and discussed.

For the first time the occurrence of the leopard (Panthera pardus) in the horizon of Rixdorf (Early Weichsel, Late Pleistocene) of Berlin-Brandenburg is proven. The find is represented by a fragment of a humerus from Niederlehme near Königs Wusterhausen. This is the most northern occurrence of the leopard in Middle Europe until now.
\end{abstract}

Key words: Panthera, humerus, late Pleistocene, Berlin, Germany.

\section{Einleitung}

Wenig außerhalb der Berliner Stadtgrenze im Südosten liegt nahe bei Königs Wusterhausen der kleine Ort Niederlehme. Seit mehr als hundert Jahren werden hier Sande abgebaut, glaziofluviatile weichselzeitliche Schmelzwassersande und -kiese, die im Liegenden und meist auch im Hangenden von Geschiebemergel begrenzt sind und sich zwischen der südlichen Eisrandlage von Brandenburg und der nördlichen von Frankfurt (Oder) ausbreiten. Das Hochflächengebiet um Niederlehme liegt im Süden des Berliner Urstromtals. Über dem liegenden Geschiebemergel sind steinige Kiessande $(1-2 \mathrm{~m})$ als Fundschicht der Rixdorfer Säugetierfauna abgelagert, darüber folgen dann etwa $20 \mathrm{~m}$ Sande. Der untere Geschiebemergel ist nach Cepek $(1975,1986)$ elsterzeitlich, der obere wird als W 1-Grundmoräne (Brandenburger Stadium) angesprochen. Die Zähne und Knochen wurden von den
Schmelzgewässern aus oberflächennahen, unterschiedlich alten Ablagerungen ausgespült und von Kiessanden erneut eingebettet. Nachgewiesen sind bisher in Niederlehme 19 Säugetierarten:

Rodentia: Castor fiber. Carnivora: Panthera spelaea - Crocuta crocuta spelaea - Canis lupus Alopex lagopus - Ursus arctos/spelaeus - Gulo gulo. Proboscidea: Mammuthus primigenius. Perissodactyla: Coelodonta antiquitatis - Dicerorhinus kirchbergensis - Equus cf. przewalskii Equus (Hemionus) hemionus. Artiodactyla: Cervus elaphus - Dama dama - Alces alces - Megaloceros giganteus - Rangifer tarandus - Bison priscus - Ovibos moschatus.

Es sind teils warmzeitliche (Dicerorhinus kirchbergensis), teils nördlich kaltzeitliche Formen (Alopex, Gulo, Mammuthus, Coelodonta, Rangifer, Ovibos) und solche, die einer einheimischen gemäßigten, vom Süden zugewanderten Fauna angehören (z. B. Castor, Cervus, Dama, Alces, Megaloceros). Nicht durch Sammlungsmaterial aus

\footnotetext{
${ }^{1}$ Institut für Paläontologie, Museum für Naturkunde, Invalidenstraße 43, D-10115 Berlin, Germany. Erhalten November 1999, angenommen Mai 2000
} 
Niederlehme belegt sind die Arten Equus (Asinus) hydruntinus und Elephas antiquus. Fossilreste wurden in Niederlehme Ende des vorigen Jahrhunderts besonders von Geologen der PreuBischen Geologischen Landesanstalt, von Pfarrer Domnick (Niederlehme) und später bis 1962 von Dietrich (Geologisch-Paläontologisches Institut und Museum im Museum für Naturkunde) aufgesammelt. Altermäßig stufte Dietrich $(1932,1968)$ die Rixdorfer Fauna in ein Frühweichsel-Interstadial ein, welches vor dem Brandenburger Vorstoß gewesen sein könnte. Während dieser interstadialen Zeitphase wurde die einheimische gemäßigte Fauna mit einer arktischen Faunenkomponente zusammengeführt. $\mathrm{Ob}$ wirklich ein Interstadial oder nur ein glaziofluviatiler Aufarbeitungshorizont der letzten Kaltzeit vorliegt. kann noch nicht endgültig entschieden werden (Heinrich 1992). Die Lokalität Niederlehme wurde inzwischen als Parastratotypus des "Rixdorfer Horizontes" bestimmt, weil die ursprüngliche Typuslokalität Rixdorf seit langem nicht mehr zugänglich ist. Rixdorf ist heute ein Teil des Stadtgebietes von Berlin-Neukölln. Aus der ehemaligen Kiesgrube wurde der Körner-Park. benannt nach dem damaligen Besitzer. Eine Gedenktafel weist heute auf die Fundgrube hin.

\section{Beschreibung}

Den 19 Faunenelementen von Niederlehme kann jetzt ein weiteres hinzugefügt werden. Vor einigen Jahren brachte uns ein Privatsammler ein rechtes distales Humerus-Fragment. welches ohne eingehende Untersuchung wegen nicht zur Hand gewesener Vergleichsmaterialien und wegen der Größe als starker Wolf (Canis lupus) vorbehaltlich in die Sammlung aufgenommen wurde. Nach nunmehr näherer vergleichender Betrachtung ist das Stück als Leopard anzusprechen:

\section{Panthera pardus (Linnaeus, 1758)}

Material: MB.Ma.39256 des Gruppenkataloges im Institut für Paläontologie. Museum für Naturkunde der HumboldtUniversität zu Berlin. Humerus dexter, distale Hälfte (Abb. 1A. 2A).

Schaft und Gelenkende sind gut erhalten. Die Farbe ist ein sehr heller Braunton. Besonders charakteristisch für das Fragment ist das an der medialen Seite über dem Gelenk liegende spaltförmige Foramen supracondylicum (= entepicondylicum), welches unter den Carnivoren bei
Katzen (Felidae) und Mardern (Mustelidae) ausgebildet ist. Der größte infrage kommende Mustelide ist Gulo (Vielfraß), er scheidet aber wegen des zu kleinen Oberarmknochens von vornherein aus. Nach den Größenproportionen kommt nur ein Leopard in die engere Wahl; der Löwe ist beträchtlich größer; der Luchs (Lynx lynx) ist kleiner (Abb. 1C, 2C), nur die größten Luchs-Exemplare können unter Umständen Leopardengröße erreichen (Tab. 1). Am Humerus des Luchses, zugrunde liegt ein rezentes Exemplar aus dem Kaukasus (ZM. 58132, Abb. 1C, 2C). fällt auf, dass das Foramen stärker in die Länge gezogen ist, weiter distal reicht als beim Leoparden und dass die Breite der Trochlea im Verhältnis zur distalen Humerus-Breite beim Luchs geringer ist. Der Luchs-Humerus aus dem eemzeitlichen Travertin von Weimar (IQW 1972/ 13455; Wei. 515) wird von Hemmer (1984) einem sehr starken männlichen Tier zugeschrieben. Nach den Zeichnungen hat das Foramen su-

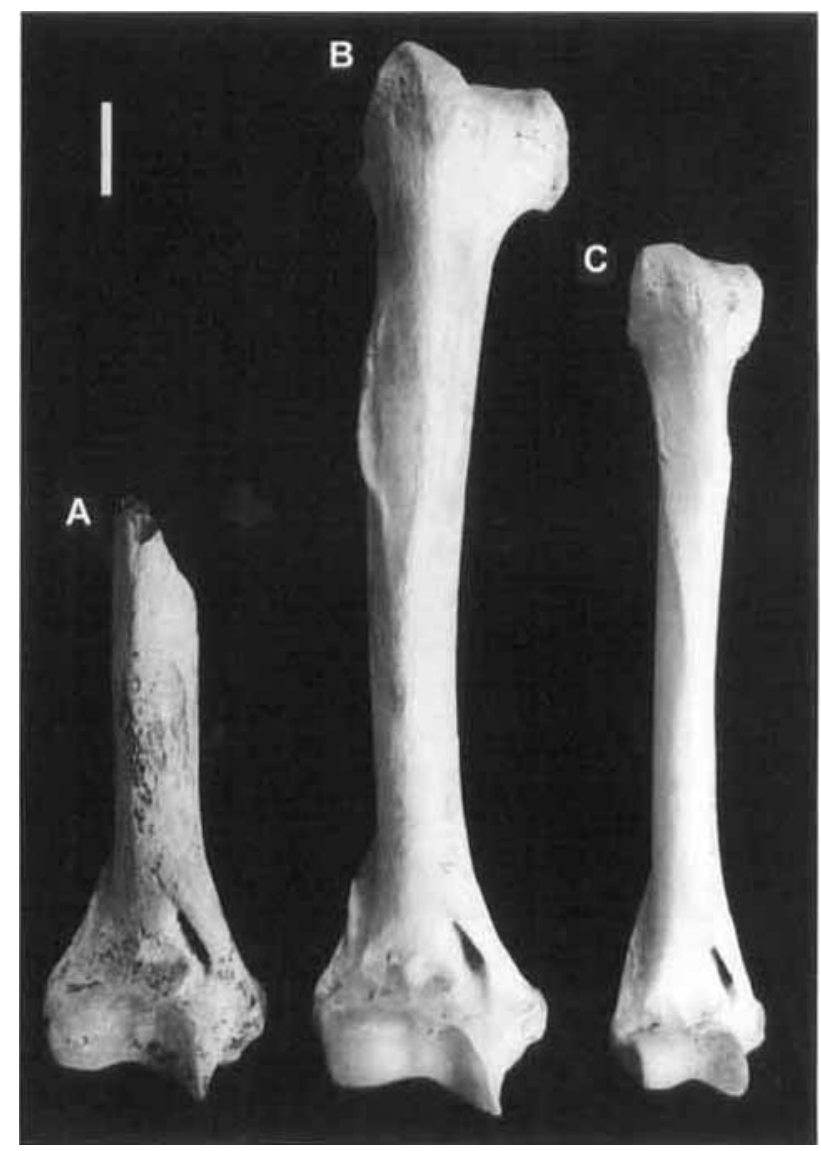

Abb. 1. Humerus dexter, Kranialansicht. A, Panthera pardus aus dem Jungpleistozän von Niederlehme, Deutschland. B, Rezentes Exemplar von "Persien". C, Lynx lynx aus dem Kaukasus

Fig. 1. Cranial views of humerus dexter of certain felids. A, Panthera pardus from the upper Pleistocene of Niederlehme, Germany. B. Recent specimen of "Persia". C, Lynx lynx from the Caucasus 
pracondylicum nur von der Seite gesehen einen verlängerten Spalt. Auch die größten Humeri des Luchses scheinen also nicht die Größe derjenigen des Leoparden zu erreichen. Verglichen mit dem Humerus eines männlichen Leoparden aus "Persien" (Museum für Naturkunde Berlin, Institut für Systematische Zoologie, ZM.16394, Abb. 1B, 2B) lässt sich feststellen, dass weder morphologisch noch metrisch, bis auf äußerst geringfügige individuelle Ungleichheiten, irgendwelche Abweichungen zu verzeichnen sind. Die Humeri des würmzeitlichen Leoparden von Vraona in Griechenland (Panthera pardus vraonensis Nagel, 1999) zeigen Mittelwerte, die geringfügig über den Maßen des Fundstückes liegen.

\section{Die Verbreitung des Leoparden im Pleistozän Europas}

Nach unseren bisherigen Kenntnissen ist das Vorkommen pleistozäner Leoparden in Europa

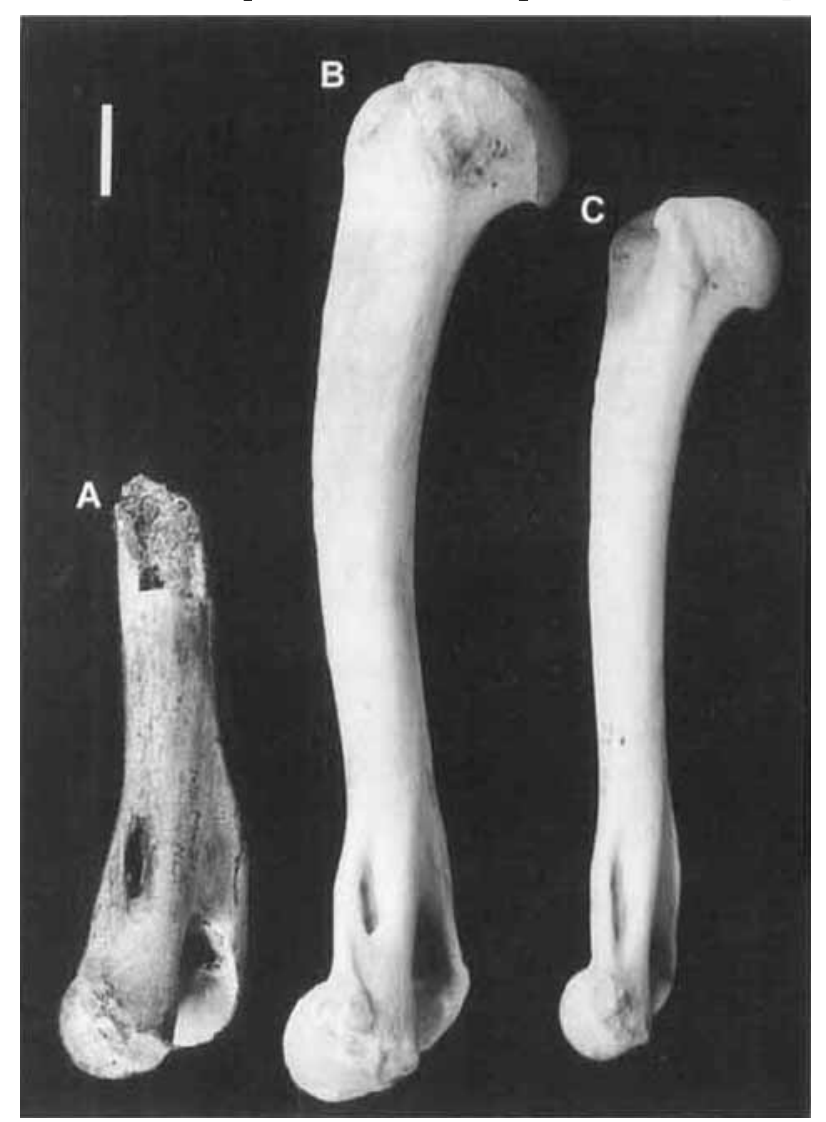

Abb. 2. Humerus dexter, Medialansicht. A, Panthera pardus aus dem Jungpleistozän von Niederlehme, Deutschland. B, Rezentes Exemplar von "Persien". C, Lynx lynx aus dem Kaukasus

Fig. 2. Medial views of humerus dexter of certain felids. $\mathbf{A}$, Panthera pardus from the upper Pleistocene of Niederlehme, Germany. B, Recent specimen of "Persia". C, Lynx lynx from the Caucasus nicht außergewöhnlich. Die Geschichte des Leoparden in Europa beginnt spätestens im obersten Pliozän (oberes Villafranchium) von Süd- und Westeuropa (z. B. Olivola, Valdarno, Tegelen), hier kommt eine Pantherkatze, Panthera gombaszoegensis (Kretzoi 1938), vor, die Merkmale des Jaguars und der primitiven Löwen zeigt und als Stammform von Jaguar, Leopard und Löwe betrachtet wird (Hemmer 1971a, Hemmer \& Schütt 1969). Diese Panther-Form soll bis ins obere Biharium (Cromer/ Elster) reichen (z. B. in Gombaszoeg, Voigtstedt, Süssenborn), wobei allerdings jüngere, nicht genau determinierbare Funde zum Leoparden (Panthera pardus) gehören könnten. Eine Unterscheidung beider Formen ist schwierig und wohl nicht in jedem Fall möglich. Kotsakis \& Palombo (1979) gehen deshalb soweit, dass sie Panthera gombaszoegensis zu einem Synonym von Panthera pardus erklären. Nach Hemmer (1971b) lebten in Gombaszoeg, Stranska Skala und Mosbach P. gombaszoegensis und $P$. pardus möglicherweise nebeneinander. Im Toringium (Mittel- - Jungpleistozän) ist $P$. gombaszoegensis verschwunden, während der Leopard aus dem Holstein ('Felis lunellensis' von Lunel-Viel, Hemmer 1972) bis ans Ende der Weichsel-Kaltzeit vorkommt. Neben dem neuen Fund aus Niederlehme sind auch die Materialien aus der Baumannshöhle von Rübeland/Harz (Schütt 1968, 1969b) frühweichselzeitlich. Der Leopard von Rübeland und etliche andere Funde, z. B. aus der Zoolithenhöhle von Burgaillenreuth (Oberfranken), wurden früher als Felis antiqua Cuvier, 1835 bezeichnet. Rübeland war bisher der nördlichste Fundpunkt in Mitteleuropa, nunmehr ist es also Berlin-Niederlehme/ Brandenburg. Der nördlichste Fundpunkt Europas liegt in Mittelengland, in Creswell/ Derby (Schmid 1940), noch etwas über der Breite von Berlin. Das Alter der Höhlenfundschicht ist nach Stuart (1974) Devensian (spätes Weichsel). Eine von den jüngsten europäischen Fundstellen liegt bei Saalfeld/Thüringen. Die Fundschichten der ehemaligen Höhle "Teufelsbrücke" gehören ins Spätweichsel (Magdalenien, Ende Dryas-2, nach Musil 1980a).

Die geographische Verbreitung des Leoparden in Europa (Abb. 3: Kartenskizze mit tabellarischer Erklärung) reicht im Süden von Gibraltar bis Griechenland, als östlichster Fundpunkt kann Kudaro (Süd-Kaukasus) noch mit einbezogen werden. Die nördlichsten Fundpunkte liegen in England und Deutschland. Kurz hingewiesen sei noch auf neuere Fundpunkte in Frankreich (Argant 1991) und Griechenland (Tsoukala 1999). 


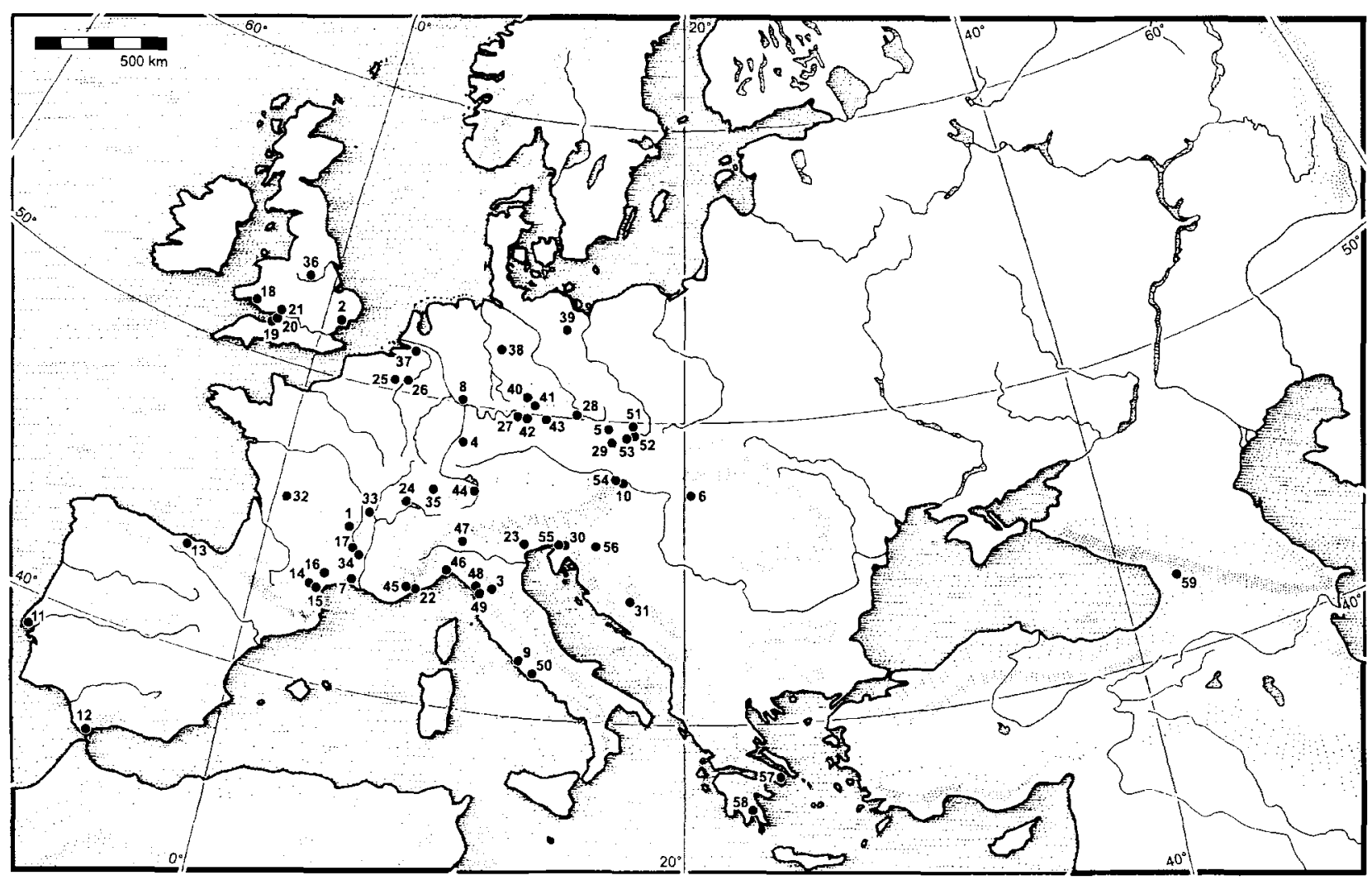

Abb. 3. Verbreitung des Leoparden (Panthera pardus) im Pleistozän Europas

Altpleistozän (1-6): 1. Frankreich: Perrieri Puy-de-Dôme (Schmid 1940). 2. England: Newborn (Schmid 1940). 3, Italien: Valdarno (Schmid 1940). 4. Deutschland: Mauer bei Heidelberg. Cromer-Warmzeit (Schütt 1969a). 5, Tschechien: Stránska Skála bei Brünn (Thenius 1972). 6. Ungarn: Gombaszög (Kretzoi 1938).

Mittelpleistozän (7-10): 7. Frankreich: Lunel-Viel. Holstein-Warmzeit (Hemmer 1972). 8. Deutschland: Mosbach/Wiesbaden (Hemmer \& Schütt 1969). 9. Italien: Rom/ Monte Sacro (Kotsakis \& Palombo 1979). 10. Österreich: Hundsheim (Schmid $1940)$.

Mittel- oder Jungpleistozän (11-31): 11. Portugal: Furninha bei Lissabon (Schmid 1940). 12, Spanien: Gibraltar, Genista-Höhle (Schmid 1940). 13. Spanien: Hornos bei Santander (Schmid 1940). 14. Frankreich: Höhle von Massat/ Ariège (Schmid 1940). 15. Frankreich: Höhle von Lherme bei Foix/ Ariège (Schmid 1940). 16. Frankreich: Cannes/ Aude (Schmid 1940). 17, Frankreich: Nabrigas/ Lozère (Schmid 1940). 18. England: Spritsail Tor/ Gower ? (Schmid 1940). 19, England: Banwell/ Mendip Hills (Schmid 1940). 20. England: Bleadon/ Mendip Hills (Schmid 1940). 21. England: Hutton ?/ Sandford Hill (Schmid 1940). 22. Italien: Nice (Schmid 1940). 23. Italien: Romagnano (Schmid 1940). 24. Frankreich: Puits de Balmes/ Rhônebecken (Schmid 1940). 25. Belgien: Höhle von Goffontaine/Liège (Schmid 1940). 26. Belgien: Höhle von Chokier/Liège (Schmid 1940). 27, Deutschland: Zoolithenhöhle von Burggaillenreuth/ Oberfranken (Schmid 1940). 28, Tschechien: Tuska Mastal bei Beroun/ Böhmen (Schmid 1940). 29. Tschechien: Kostelik/ Mokrá-Wald (Schmid 1940). 30. Slowenien: (Pavsic \& Turk 1989). 31. Bosnien-Herzegowina: Vjetrenica-Höhle. Popovo Polje (Malez \& Pepeonik 1970).

Jungpleistozän (32-59): 32. Frankreich: Jaurens-Höhle/ Corrèze. Würm (Ballesio 1980). 33. Frankreich: Bourgogne (Verzé, Blanot 2. Etrigny) (Argant 1991). 34. Frankreich: Mialet/Gard. Würm (Schmid 1940). 35, Schweiz: Höhle von Cotencher/ Neuchâtel (Schmid 1940). 36. England: Creswell (Derby). Spät-Weichsel (Schmid 1940. Stuart 1974). 37, Niederlande: Meuse und Waal (Bosscha Erdbrink 1986). 38. Deutschland: Baumannshöhle in Rübeland/ Harz, Früh-Weichsel (Schütt 1968, 1969b). 39. Deutschland: Niederlehme/ Land Brandenburg bei Berlin. Früh-Weichsel (MS.). 40, Deutschland: Taubach/ Thüringen, Eem-Warmzeit (Schmid 1949). 41. Deutschland: Teufelsbrücke bei Saalfeld/ Thüringen. Spät-Weichsel (Musil 1980). 42. Deutschland: Rabenstein / Oberfranken (Hemmer 1971a). 43. Deutschland: Petershöhle bei Velden/ Oberfranken ?EemWarmzeit (Schmid 1940). 44. Schweiz: Wildkirchli bei Appenzell. Eem-Warmzeit (Schmid 1940). 45. Italien: Mars-Höhle bei Vence/Liguria (Schmid 1940). 46. Italien: Fate-Höhle/ Liguria (Schmid 1940). 47. Italien: Zandobbio bei Bérgamo, Riß/Würm (Vialli 1957). 48. Italien: Equi-Grotte bei Fivizzano/ Alpi Apuane (Del Campana 1954). 49, Italien: Höhle bei Cucigliana/ Monti Pisani (Schmid 1940). 50, Italy: S. Agostino bei Gaeta (Tozzi 1970). 51, Tschechien: Schwedentischgrotte nahe Brno/ Mähren (Schmid 1940). 52. Tschechien: Predmost bei Prerau. Würm (Schmid 1940). 53, Tschechien: Vypustek-Höhle bei Kiritein/ Mähren. Würm (Schmid 1940). 54. Österreich: Merkenstein/ Niederösterreich, südlich Wien (v.Wettstein \& Mühlhofer 1938). 55. Italien: Pocala-Höhle. Aurisina bei Triest (Anelli 1954). 56. Kroatien: Veternica-Höhle bei Zagreb (Musil 1980). 57. Griechenland: Vraona bei Athen. Spätwürm (Nagel 1999). 58. Griechenland: Apidima-Höhle, Lakonia, Süd-Peloponnes (Tsoukala 1999). 59. Georgien: Kudaro-Höhle. Süd-Kaukasus (Verestchagin 1959).

Fig. 3. Distribution of the leopard Panthera pardus in the Pleistocene of Europe.

Early Pleistocene (1-6): France: Perrier/ Puy-de-Dôme (Schmid 1940). 2. England: Newborn (Schmid 1940). 3, Italy: Valdarno (Schmid 1940). 4. Germany: Mauer near Heidelberg. Cromer Interglacial (Schütt 1969a). 5, Czechia: Stránská Skála near Brno (Thenius 1972). 6. Hungary: Gombaszög (Kretzoi 1938).

Middle Pleistocene (7-10): 7. France: Lunel-Viel. Holstein Interglacial (Hemmer 1972), 8, Germany: Mosbach/Wiesbaden (Hemmer \& Schütt 1969). 9. Italy: Rome/ Monte Sacro (Kotsakis \& Palombo 1979). 10. Austria: Hundshcim (Schmid 1940). Middle or Late Pleistocene (11-31): 11. Portugal: Furninha near Lisbon (Schmid 1940). 12. Spain: Gibraltar, cave of Genista 
Weitere meist ältere Fundorte (Schmid 1940) sind bekannt aus Portugal, Spanien, Frankreich, Belgien, England, Italien, der Schweiz, Österreich, Tschechien und Ungarn.

\section{Formenvielfalt, Umwelt, Fundmaterial}

Die im Laufe des Pleistozäns in Europa auftretenden Leoparden gehören wahrscheinlich vier unterschiedlichen Verwandtschaftsgruppen an. Die südeuropäischen Formen des älteren Pleistozäns sind vom cromerzeitlichen Leoparden (Panthera pardus sickenbergi) aus Mauer zu trennen. Die Formen des mittleren (Mosbach) bis jüngeren Pleistozäns (Taubach; Schmid 1949) bilden eine Gruppe, die sich von den jungpleistozänen bis holozänen Formen Osteuropas unterscheidet. Der würmzeitliche Leopard Frankreichs (Jaurens-Höhle, Corrèze) kann der MosbachTaubach-Gruppe zugeordnet werden (Ballesio 1980). Die jungpleistozänen östlichen Formen, zu denen die Leoparden von Rübeland, Saalfeld und Niederlehme gehören, lösten wohl die ältere Gruppe (Mosbach-Taubach) ab, die wahrscheinlich Beziehungen $\mathrm{zu}$ afrikanischen Leoparden hatte. Nach Hemmer (1971) können die Leoparden von Rübeland mit den rezenten des Kaukasus verglichen werden. Von den etwa 24 Unterarten des heutigen Leoparden in Asien und Afrika sind $3 \mathrm{im}$ kaukasischen Raum beheimatet. Zukowsky (1959) unterscheidet Panthera pardus saxicolor, den persischen Panther (der zum Vergleich vorliegende rezente Humerus aus dem Museum für Naturkunde Berlin, Institut für Systematische Zoologie, stammt wohl von dieser Subspezies) der Waldgebiete südlich des Kaspischen Meeres, die kleinere Rasse P. pardus tullia$n a$, verbreitet von Aserbaidschan über Armenien bis Kleinasien und die größere Rasse $P$. pardus ciscaucasica vom Kaukasus und dem nördlich vorgelagerten Flussgebiet des Kuban.

Das gemäßigte Klima der Frühweichselzeit, ja sogar das kühl-gemäßigte bis subarktische der späten Weichselzeit (Saalfeld/Teufelsbrücke) war für den Leoparden nicht ungewöhnlich. Von den heutigen Formen wissen wir, dass sie vorzüglich an sehr unterschiedliche klimatische Lagen angepasst sein können und hohe Sommertemperaturen, sehr niedrige Wintertemperaturen, Trockenheit und Feuchtigkeit gut vertragen, solange der Bestand an Beutetieren gesichert ist. Wie z. B. Biber und Rothirsch auf Baumbestand in der jungpleistozänen Landschaft des Berliner Raumes hindeuten, lässt auch der Leopard vermuten, dass Bäume vorhanden waren, auf denen er, ähnlich wie seine heutigen Nachkommen, Beute vor dem Zugriff der Löwen und Hyänen in Sicherheit brachte. Baum- und Strauchbestand wird auch für die Spätweichselzeit bei Saalfeld angenommen, wo licht bewaldete Talhänge an die Kaltsteppen der Hochfläche angrenzten.

Da ein Vorkommen des Leoparden nicht ohne weiteres auf das Klima schließen lässt, können Vermutungen über eine genauere altersmäßige Einstufung der Höhlenfunde von Gibraltar, Slowenien und Bosnien-Herzegowina (siehe Verbreitungstabelle) nicht geäußert werden.

(Schmid 1940). 13, Spain: Honos near Santander (Schmid 1940). 14, France: Cave of Massat/ Ariège (Schmid 1940). 15, France: Cave of Lherme near Foix/ Ariège (Schmid 1940). 16, France: Cannes/ Aude (Schmid 1940). 17, France: Nabrigas/ Lozère (Schmid 1940). 18, England: Spritsail Tor/ Gower? (Schmid 1940). 19, England: Banwell/ Mendip Hills (Schmid 1940). 20. England: Bleadon/ Mendip Hills (Schmid 1940). 21, England: Hutton ?/ Sandford Hill (Schmid 1940). 22, Italy: Nice (Schmid 1940). 23, Italy: Romagnano (Schmid 1940). 24, France: Puits de Balmes/ Basin of Rhône (Schmid 1940). 25, Belgium: Cave of Goffontaine/ Liège (Schmid 1940). 26, Belgium: Cave of Chokier/ Liège (Schmid 1940). 27, Germany: Zoolithic Cave of Burggaillenreuth/ Bavaria (Schmid 1940). 28, Czechia: Tuska Mastal near Beroun/ Bohemia (Schmid 1940). 29, Czechia: Kostelik/ Forest of Mokrá (Schmid 1940). 30, Slovenia: (Pavsic \& Turk 1989). 31, Bosnia-Herzegovina: Cave of Vjetrenica/ Popovo Polje (Malez \& Pepeonik 1970).

Late Pleistocene (32-59): 32, France: Cave of Jaurens/ Corrèze, Würm (Ballesio 1980). 33, France: Bourgogne (Verzé, Blanot 2, Etrigny (Argant 1991). 34, France: Mialet/ Gard, Würm (Schmid 1940). 35, Switzerland: Cave of Cotencher/ Neuchâtel (Schmid 1940). 36, England: Creswell/ Derby, Late Weichsel (Schmid 1940, Stuart 1974). 37, Netherlands: Meuse and Waal (Bosscha Erdbrink 1986). 38, Germany: Cave of Baumann in Rübeland/ Harz, early Weichsel (Schütt 1968, 1969b). 39, Germany: Niederlehme/ Brandenburg, near Berlin, early Weichsel. 40, Germany: Taubach/ Thuringia, Eem Interglacial (Schmid 1949). 41, Germany: Teufelsbrücke near Saalfeld/ Thuringia, Late Weichsel (Musil 1980). 42, Germany: Rabenstein/ Bavaria (Hemmer 1971a). 43, Germany: Petershöhle near Velden/ Bavaria, ? Eem Interglacial (Schmid 1940). 44, Switzerland: Wildkirchli near Appenzell, Eem Interglacial (Schmid 1940). 45, Italy: Cave of Mars near Vence/ Liguria (Schmid 1940). 46, Italy: Cave of Fate/ Liguria (Schmid 1940). 47, Italy: Zandobbio near Bérgamo, Riß/Würm (Vialli 1957). 48, Italy: Cave of Equi near Fivizzano/ Alpi Apuane (Del Campana 1954). 49, Italy: Cave near Cucigliana/ Monti Pisani (Schmid 1940). 50, Italy: S. Agostino near Gaeta (Tozzi 1970). 51, Czechia: Cave of Schwedentisch near Brno/ Moravia (Schmid 1940). 52, Czechia: Predmost near Prerau, Würm (Schmid 1940). 53, Czechia: Cave of Vypustek near Kirirein/ Moravia, Würm (Schmid 1940). 54, Austria: Merkenstein/ Niederösterreich, near Wien (v. Wettstein \& Mühlhofer 1938). 55, Italy: Cave of Pocala, Aurisina near Triest (Anelli 1954). 56, Croatia: Cave of Veternica near Zagreb (Musil 1980). 57, Greece: Cave of Vraona/ Athen, Late Würm (Nagel 1999). 58, Greece: Cave of Apidima/ Lakonia/ Süd-Peloponnes (Tsoukala 1999). 59, Georgia: Cave of Kudaro/ South-Caucasus (Verestchagin 1959). 
Táb. 1

Humerus

\begin{tabular}{|c|c|c|c|c|c|c|}
\hline \multirow[b]{2}{*}{ Maße (in mm) } & \multicolumn{4}{|c|}{ Panthera pardus } & \multicolumn{2}{|c|}{ Lynx lynx } \\
\hline & Niederlehme & Rübeland & Vraona. n: 5 & ..Persien" & Weimar & Kaukasus \\
\hline Max. Länge & - & 220.0 & _- & 221.0 & 210,0 & 184,5 \\
\hline Min. Breite. dist. Diaphyse & 18.5 & - & $13.8-24.6$ & 18,2 & - & 14,0 \\
\hline Max. Breite. dist. & 49.0 & 50,2 & - & 52.6 & 42,0 & 38,0 \\
\hline Max. Dicke. dist.. kraniokaudal & 30.0 & - & - & 32.0 & 23,5 & 21,0 \\
\hline Max. Breite. Trochlea & 37.0 & - & $25.2-46.3$ & 40.0 & 27,5 & 26,0 \\
\hline Trochlea-Breite / dist. Breite in \% & 75.5 & - & - & 76.0 & 65,5 & 68.4 \\
\hline
\end{tabular}

Anmerkung: Rübeland (Schütt 1968): "Persien". rezent. masculin. Museum für Naturkunde Berlin, Institut für Systematische Zoologie. ZM.16394: Weimar (Hemmer 1984): Kaukasus, rezent. Museum für Naturkunde Berlin, Institut für Systematische Zoologie. ZM.58132. Das Exemplar von "Persien" scheint zu den größeren Formen zu gehören wie einige vergleichende Messungen an afrikanischen Rohskeletten vermuten lassen.

Trotz einer zeitlich und räumlich ausgedehnten Verbreitung des Leoparden im europäischen Pleistozän sind Funde an den wenigen Lokalitäten selten. Nur hin und wieder haben wir von einer Fundstelle einen Kiefer, einen Zahn oder ein Knochenfragment wie das vorliegende. Skelettfunde wurden bisher nur zwei angezeigt. Das erste Skelett lag im Löß von Mosbach (Schmid 1940) und das zweite in der Vjetrenica-Höhle/ Herzegovina (Malez \& Pepeonik 1970). Beide sind nicht oder wenig eingehend bearbeitet worden und heute wohl schon nicht mehr vorhanden. Eine Erklärung zur Seltenheit der Funde kann der Hinweis sein, dass der Leopard außerhalb der Brunstzeit ein Einzelgänger ist. Auch der pleistozäne Löwe ist nicht allzu häufig, aber immerhin gibt es so viel Material, dass statistische Bearbeitungen im Gegensatz zum Leoparden-Material möglich sind.

Kaum untersucht wurde bisher das postkraniale Skelett des fossilen Leoparden, Vergleiche mit rezentem Material stehen noch aus und sind erschwert, weil Letzteres in Sammlungen (Rohskelette) und in der Literatur nicht oder ungenügend erschlossen ist. Überhaupt fehlen weitgehend grundlegende Arbeiten zur Skelettmorphologie der rezenten Pantherkatzen. z. B. auch Messungen einzelner Skelettelemente, die für Vergleiche wichtig wären (ausgenommen Hemmer \& Schütt 1970, Nagel 1999).

Fundiertere Aussagen über Exemplare wie das vorgestellte Humerusfragment und über die $\mathbf{Z u}$ ordnung zu einer bestimmten Formengruppe sind jedoch kaum jemals möglich.

\section{Danksagung}

Die Fotos wurden von Waltraud Harre (Institut für Paläontologie, Museum für Naturkunde Berlin) angefertigt.

\section{Literatur}

Anelli. F. 1954. Contributo alla conoscenza della fauna diluviale della caverna Pocala di Aurisina (Trieste). - Memorie Descrittive della Carta Geologica d'Italia 11: 5-57.

Argant. A. 1991. Carnivores Quaternaires de Bourgogne. Documents des labouratoires de Géologie, Lyon 115: 1-301.

Ballesio. R. 1980. Le gisement Pléistocène supérieur de la grotte de Jaurens à Nespouls, Corrèze, France: les carnivores (Mammalia. Carnivora). 2. Felidae. - Nouvelles Archives du Muséum d'Histoire Naturelle de Lyon 18: 61-102.

Bosscha Erdbrink. D. P. 1986. Lions, leopards and a hyaena from deposits along the Meuse and the Waal. - Koninklijke Nederlandse Akademie van Wetenschappen Amsterdam (Palaeontologie, Geologie, Physica, Chemic) 89(1): $1-13$

Cepek. A. G. 1975. Zur geologisch-stratigraphischen Interpretation des ..Rixdorfer Horizontes". In Exkursionsführer zur Jubiläumstagung .,100 Jahre Glazialtheorie im Gebiet der skandinavischen Vereisungen". Gesellschaft für Geologische Wissenschaften der DDR: 17-21.

- 1986. Schichtenfolge und Position des ..Rixdorfer Horizontes“. In Kurzreferate und Exkursionsführer „25 Jahre AK Quartärgeologie“. Gesellschaft für Geologische Wissenschaften der DDR: 19-21, Berlin.

Del Campana. D. 1954. Carnivori quaternari della Tecchia e della caverna di Equi nelle Alpi Apuane (Mustelidi, Canidi. Felidi). - Palaeontographia Italica 44, Palaeontologia ed ecologia del Quaternario 2 (1947-51): 1-42.

Dietrich. W. O. 1932. Über den Rixdorfer Horizont im Berliner Diluvium. - Zeitschrift der deutschen geologischen Gesellschaft 84(4): 193-221.

- 1968. Fossile Löwen im europäischen und afrikanischen Plcistozän. - Paläontologische Abhandlungen A, III(2): $323-366$. Berlin.

Heinrich. W.-D. 1992. .Rixdorfer Horizont" von Niederlehme bei Königs Wusterhausen. - Exkursionsführer zur 62. Jahrestagung der Paläontologischen Gesellschaft in Berlin. 57 Seiten.

Hemmer. H. 1971a. Zur Charakterisierung und stratigraphischen Bedeutung von Panthera gombaszoegensis (KRETZOI, 1938). - Neues Jahrbuch für Geologie. Paläontologie. Monatshefte 12: 701-711.

- 1971b. Zur Kenntnis pleistozäner mitteleuropäischer Leoparden (Panthera pardus). - Neues Jahrbuch für Geologic. Paläontologie. Abhandlungen 138: 15-36.

- 1972. Zur systematischen Stellung von Jansofelis vaufreyi BONIFAY 1971 und Felis lunellensis BONIFEY 1971 aus dem Pleistozän Südfrankreichs (Carnivora, Felidae). Neues Jahrbuch für Geologie, Paläontologie, Monatshefte 4: $215-223$. 
- 1984. Die Carnivoren aus den Travertinen von Weimar. Quartärpaläontologie 5: 409-425.

Hemmer, H. \& Schütt, G. 1969. Ein Unterkiefer von Panthera gombaszoegensis (KRETZOI, 1938) aus den Mosbacher Sanden. - Mainzer Naturwissenschaftliches Archiv 8: 90-101.

- 1970. Körpergröße und Extremitätenmaße ältest- und altpleistozäner europäischer Pantherkatzen (Genus Panthera). - Mainzer Naturwissenschaftliches Archiv 9: 132-146.

Kotsakis, T. \& Palombo, M. R. 1979. Un cranio di Panthera pardus (L.) del Pleistocene medio superiore di Monte Sacro (Roma). - Geologica Romana 18: 137-155.

Kretzoi, M. 1938. Die Raubtiere von Gombaszög nebst einer Übersicht der Gesamtfauna. - Annales historico-naturales Musei Nationalis Hungarici, Pars Mineralogica, Geologica, Palaeontologica 31: 88-157.

Malez, M. \& Pepeonik, Z. 1970. Entdeckung des ganzen Skelettes eines fossilen Leoparden in der Vjetrenica-Höhle auf dem Popovo Polje (Herzegovina). - Akademiski Savet FNRJ Bulletin scientifique A 14(5/6): 144-145, Zagreb.

Musil, R. 1980a. Die Großsäuger und Vögel der Teufelsbrücke. In Feustel, R. (ed.) Magdalenienstation Teufelsbrücke. II: Paläontologischer Teil. - Weimarer Monographien zur Ur- und Frühgeschichte 3: 5-59.

- 1980b. Ursus spelaeus - Der Höhlenbär I. - Weimarer Monographien zur Ur- und Frühgeschichte 2: 4-94.

Nagel, D. 1999. Panthera pardus vraonensis n. ssp., a new leopard from the Pleistocene of Vraona/ Greece. - Neues Jahrbuch für Geologie, Paläontologie, Monatshefte 3: $129-150$

Pavsic, J. \& Turk, I. 1989. First find of species Panthera pardus (L.) and new finds of Gulo gulo L. in Slovenia. Slovenska Akademija Znanosti in Umetnosti, Razprave 30: $129-160$.
Schmid, E. 1940. Variationsstatistische Untersuchungen am Gebiss pleistozäner und rezenter Leoparden und anderer Feliden. - Zeitschrift für Säugetierkunde 15: 1-179.

- 1949. Über einen Leopardenfund von Taubach. - Neues Jahrbuch für Mineralogie, Geologie, Paläontologie, Monatshefte B, Jg. 1949: 102-108.

Schütt, G. 1968. Ein jungpleistozäner Leopardenfund aus der Baumannshöhle bei Rübeland im Harz. - Mitteilungen des Geologischen Institutes der Technischen Hochschule Hannover 8: 102-115.

- 1969a. Panthera pardus sickenbergi n. ssp. aus den Mauerer Sanden. - Neues Jahrbuch für Geologie, Paläontologie, Monatshefte 5: 299-310.

- 1969b. Die jungpleistozäne Fauna der Höhlen bei Rübeland im Harz. - Quartär 20: 79-125.

Stuart, A. J. 1974. Pleistocene History of the British Vertebrate Fauna. - Biological Reviews 49: 225-266.

Thenius, E. 1972. Die Feliden (Carnivora) aus dem Pleistozän von Stránska Skála. - Studia Musei Moraviae Anthropos 20: 121-135.

Tozzi, C. 1970. La grotta di S. Agostino (Gaeta). - Rivista Scienze Preistoriche 25(1): 1-87.

Tsoukala, E. 1999. Quaternary large mammals from the Apidima Cave (Lakonia, South Peloponnese, Greece). Beiträge zur Paläontologie Österreichs 24: 207-229.

Vereshchagin, N. K. 1959. The mammals of the Caucasus. 704 pp., Akademie Nauk SSSR Moskva. (In Russian).

Vialli, V. 1957. I vertebrati della breccia ossifera dell interglaciale Riss-Würm di Zandobbio (Bergamo). - Atti della Società Italiana di Science Naturali 96: 51-84.

Wettstein, O. v. \& Mühlhofer, F. 1938. Die Fauna der Höhle von Merkenstein in Niederösterreich. - Archiv für Naturgeschichte, N.F. 7: 514-558.

Zukowsky, L. 1959. Persische Panther. - Zoologischer Garten (N.F.) 24: 329-344. 